\title{
A fast method for analyzing essential protein mutants in human cells
}

\author{
Frank Dietsch, Mariel Donzeau, Agnes M. Cordonnier, Etienne \\ Weiss, Bruno Chatton, and Marc Vigneron \\ Université de Strasbourg, Biotechnologie et Signalisation Cellulaire, \\ Ecole Supérieure de Biotechnologie de Strasbourg, Illkirch, France
}

BioTechniques 62:80-82 (February 2017) doi 10.2144/000114518

Keywords: human complementation assay; Cas9; pCEP4

Supplementary material for this article is available at www.BioTechniques.com/article/114518.

Here we developed a complementation method for the study of essential genes in live human cells using the CRISPR/Cas9 system. Proteins encoded by essential genes were expressed using a derivative of the pCEP4 compensating plasmid in combination with Cas9 endonuclease targeting of the chromosomal genes. We show that this strategy can be applied to essential genes, such as those coding for proliferating cell nuclear antigen (PCNA) and DNA polymerase delta subunit 2 (POLD2). As demonstrated for the PCNA protein, our method allows mutational analysis of essential protein-coding sequences in live cells.

The systematic analysis of the functions of non-essential genes has recently become achievable using the bacterial CRISPR/Cas9 endonuclease (1). Its selective guide RNA (gRNA)-directed cleavage of DNA ultimately yields indel mutations that inactivate targeted genes. When targeting a coding sequence, the encoded protein will be altered, most often yielding inactive or unstable translation products, potentially leading to visible and measurable cellular phenotypes. One limit of this approach is the required viability of the mutated cells, precluding the study of essential proteins.

To address this problem, we adapted the classical yeast complementation assay to human cells (2). Cas9-mediated inactivation of an essential gene was compensated with a derivative of the plasmid pCEP4 (Invitrogen, Paisley, UK) encoding the same protein (Figure 1A). The compensating plasmid was engineered to simultaneously express, under the control of a CMV promoter, the protein of interest together with a selectable fluorescent mCherry protein, enabling convenient detection of transfected cells. In addition, this plasmid contains a hygromycin resistance gene (Figure 1A). In the presence of this plasmid, the chromosomal sequences can be knocked out using an appropriately targeted CRISPR/ Cas9 endonuclease without killing the cell. The compensating plasmid is designed to escape the CRISPR/Cas9 endonuclease action and does not contain the Cas9-targeted sequence. This was achieved in two different ways (see below). The functions of two essential genes were tested using this system: the proliferating cell nuclear antigen (PCNA) and the DNA polymerase delta subunit 2 (POLD2) genes, which are both involved in nuclear DNA replication and cancer progression $(3,4)$.

We explored two different strategies allowing the compensating plasmid to escape the Cas 9 activity, one for PCNA and the second for POLD2, but both may be applied for any gene. The PCNA protein is quite conserved evolutionarily, as the mouse and human orthologs differ by only 8 residues ( $97 \%$ identity). However, their nucleotide coding sequences (CDS) differ by 88 out of 785 nucleotides (89\% identity). We synthesized an artificial CDS derived from a mouse cDNA mutated to encode a PCNA protein fully matching the human amino acid sequence. The homology between the artificial CDS and the human CDS was reduced as much as possible, allowing a variety of gRNAs against the endogenous human gene to be tested. In the case of $P O L D 2$, the CDS expressed from the compensating plasmid corresponds to the wild-type endogenous human cDNA. The Cas9 targets consisting of 20-bp recognition sequences were chosen to overlap splice junctions in the gene, with the cut site being located within the exonic CDS (red arrow, Figure 1A) so that the chromosomal genes are selectively inactivated, while the compensating plasmid is insensitive to Cas 9 (further details are included in the Supplementary Material).

In order to express the Cas9 protein together with the gRNA, we used two constructs (plasmids \#48138, pCas92A-GFP and \#62988, pCas9-2A-Puro; Addgene, Cambridge, MA) (5) (Figure 1A, right). HEK293T cells were co-transfected with either of these plasmids together with the compensating plasmid described above. Doubly fluorescent cells (red and green) were selected using FACS and allowed to grow (Figure 1B). Alternatively, co-transfected cells were selected by successively adding puromycin $(0.5 \mu \mathrm{g} / \mathrm{mL}$ for 48 h) and hygromycin $(0.2 \mathrm{mg} / \mathrm{mL}$ for $48 \mathrm{~h})$ to the culture medium (Figure 1C). Using both methods, expression of the compensating protein (PCNA or POLD2) by the compensating plasmid led to the growth of a large number of colonies (Figure 1, B and C, right). In contrast, essentially all of the knockout cells died 8-10 days after selection in the presence of an empty compensating plasmid (Figure 1, B and C, left). As can be seen, some clones survived the selection process; this is inherent to the Cas9 system itself. Notably, some alleles escape the

\section{METHOD SUMMARY}

Because Cas9 endonuclease-mediated knockout of an essential gene is lethal, we constructed an EBV (Epstein-Barr virus) episomal expression vector to complement the absence of the essential protein. The compensating vector restores viability following CRISPR/ Cas9-induced gene knockout, allowing a systematic mutagenesis of the essential protein of interest to define its domains and functions. 

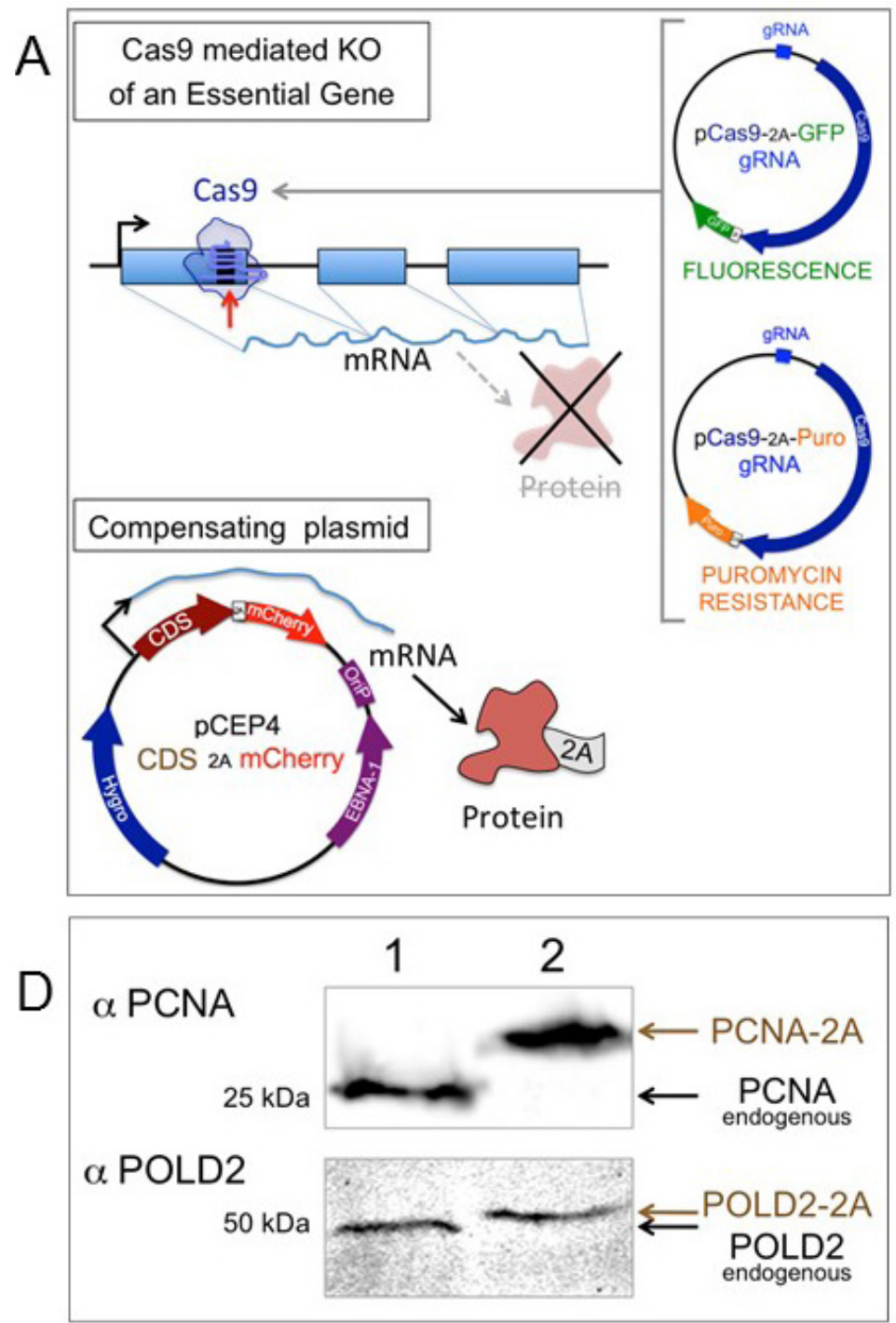
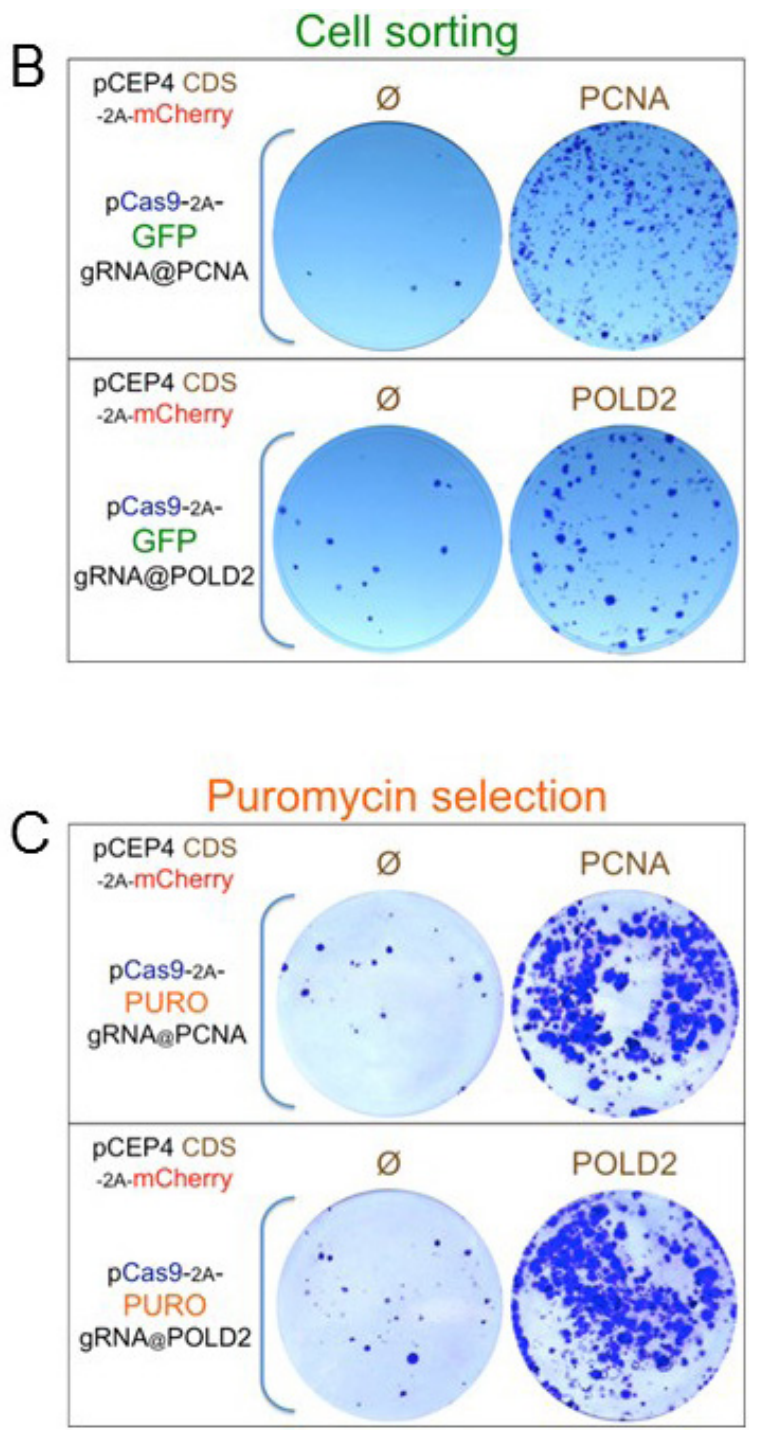

Figure 1. PCNA and POLD2 knock-out compensation in human cells. (A) Schematic representation of the strategy: An essential target gene is knocked out using a targeted Cas9 endonuclease complex (red arrow) encoded by two alternative constructs allowing either fluorescence or puromycin-resistance selection. A compensating pCEP4-derivative plasmid allows the cell to survive by expressing the compensating protein together with a mCherry fluorescent reporter protein, yielding a protein with an additional C-terminal 22-amino-acid peptide encoded by the 2A-peptide sequence. (B) HEK293T cells were co-transfected using a pCas9-2AGFP plasmid expressing a guide RNA (gRNA) targeting either the PCNA or POLD2 gene (top and bottom panels, respectively), as well as an empty pCEP4 plasmid or a pCEP4 plasmid expressing PCNA or POLD2 as indicated. Transfected cells (2500 cells total) were selected using a cell sorter and allowed to grow in 5-cm Petri dishes. After 10 days, the colonies were stained using crystal violet. (C) HEK293T cells were co-transfected using a pCas9-2A-Puro plasmid expressing a gRNA directed to either the PCNA or POLD2gene (top and bottom panels, respectively) as well as the indicated pCEP4 plasmids. Cells were selected for resistance to puromycin and hygromycin for 2 days, successively. After 12 days, the colonies were stained using crystal violet. (D) Western blot. Total cell extracts either from control HEK293T cells (1) or from selected cells (2) were electrophoresed on 9\%-11\% SDS acrylamide gels. After transfer to nitrocellulose membranes the proteins were visualized using the appropriate antibodies (Santa Cruz Biotechnology, Dallas, TX) as indicated on the left (for PCNA: PC-10, Sc-56; and for POLD2: C-20, Sc-8800).

action of the Cas9 complex, although with a very low frequency, leading to cells in which a wild-type allele can be found together with alleles exhibiting the typical Cas9-induced indel mutations (data not shown).

To verify that the endogenous protein was effectively replaced by the plasmidencoded protein, we performed western blots using total cell extracts of selected cells (Figure 1D). The endogenous PCNA and POLD2 proteins were both detectable in control HEK293T cells (Figure 1D, lane 1). In contrast, these proteins were absent in the selected compensated cells, whereas the same antibodies revealed a shifted band as expected due to the presence of the 2A peptide-encoded C-terminal extension (Figure 1D, lane 2). This result confirmed the specificity of both the Cas9 targeting and the antibody recognition. Remarkably, the expression levels of the complementing and endogenous POLD2 proteins were very similar, while expression of the compensating PCNA was 3- to 4-fold higher than that of the endogenous protein. The same results were obtained irrespective of the cell selection method (data not shown). The presence of remaining wild-type chromosomal alleles in a recently complemented cell population was undetectable by western blot (see Figure 1D, lane 2). However, after 2 months of culture without any selective pressure, the endogenous proteins were detectable again (data not shown). To avoid this drawback, one may select cellular clones that are known not to contain any wild-type alleles. Alternatively, an additional hygromycin selection quite effectively removes cells with a wild-type genomic allele from the population, and only the compensating plasmid-encoded protein remains detectable after this treatment (data not shown). 
Figure 2. Analysis of PCNA mutants. (A) Front and side views of human PCNA protein structure (1axc.pdb, RCSB Protein Data Bank) highlighting the K164 (green) and Y211 (blue) residues. (B) Clonogenic assays. One thousand cells were distributed in each well of a 6-well plate and subjected to UV irradiation $\left(254 \mathrm{~nm}, 5 \mathrm{~J} / \mathrm{m}^{2}\right)$ or left untreated. After 8 days of incubation, the cells were fixed and stained with crystal violet. The PCNA-inactivated cells expressed the wild-type (WT), K164R, or Y211F PNCA proteins from the compensating plasmids, as indicated. (C) The ratio of the number of UVsurviving colonies compared with the untreated cells in four independent experiments is shown. Standard deviation (SD) and statistical significance as determined by Student's $t$ test are shown: NS = nonsig nificant; ${ }^{* *} P<0.001$.
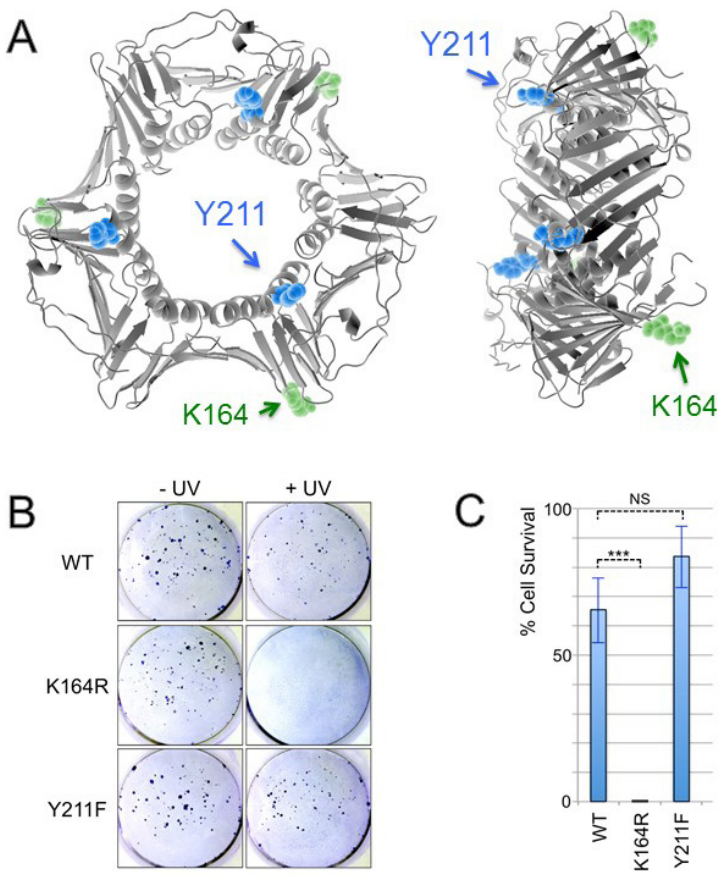

To illustrate the utility of our system for mutational analysis, we tested the complementation potential of two PCNA mutants: K164R and Y211F $(6,7)$ (Figure 2A). Both mutations impair post-translational modifications, but they did not affect the viability of the complemented cells (data not shown). The PCNA K164R mutation has been shown to impair ubiquitination of PCNA in response to UV irradiation, thereby preventing initiation of the DNA damage response (6); in contrast, Y211 phosphorylation has been shown to be involved in PCNA stability (7). We therefore performed a UV sensitivity assay on complemented cells (Figure 2B). Mild UV irradiation (254 $\mathrm{nm}$ ) efficiently killed the K164R PCNAcomplemented cells, while only moderately affecting both WT and Y211F-complemented cells. Under these conditions, the $\%$ cell survival dropped from $65 \%$ and $80 \%$ for the WT and Y211F mutants, respectively, to $0.1 \%$ for the K164R mutant, demonstrating that the UV sensitivity phenotype of the PCNA K164R mutant was detectable using our test (Figure 2C).

Using a pCEP4-compensating plasmid, we are able to compensate for the loss of essential gene functions in HEK293T cells when Cas9 is used to inactivate the corresponding chromosomal copy of the gene. This co-transfection system mimics the classical yeast complementation assay (2), and its application opens the possibility of fast and cheap mutational analysis of essential proteins in living mammalian cells in which the Epstein-Barr virus origin of replication is functional (primate/human or canine). The single-stranded oligodeoxynucleotide donor (ssODN)-mediated gene editing of any gene-including those that are essential-at its endogenous locus is an alternative technique that can be used for the same purpose. Since mutated genes remain under native expression control, this method would be preferable (8). It is, however, much more difficult technically, while our assay is quite easy to carry out.

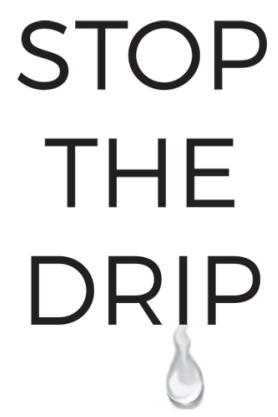

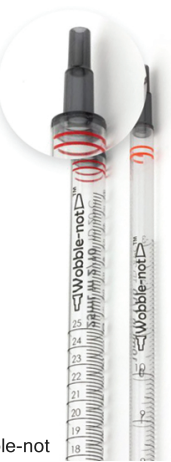

The Better Way to Pipette

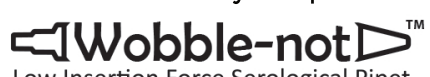

Low Insertion Force Serological Pipet

No Drip Design

2 -tiers $/ 2$ steps $=2 X$ contact in your controller $=$ better seal

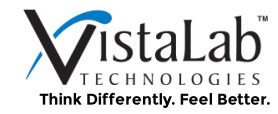

Don't mess with dripping. Get FREE Samples! 四 www.vistalab.com/wobble-not/\#get-free-sample-pack

\section{Author contributions}

F.D. performed the experiments. M.D., A.C. performed a critical analysis of the results. E.W., B.C., and M.V. conceived the assay strategy. E.W. and M.V. wrote the paper.

\section{Acknowledgments}

This work was supported by funds and/or fellowships from the Centre National de la Recherche Scientifique, the University of Strasbourg, the French Ministry of Research, the Ligue Régionale contre le Cancer (CCIR-GE), and the Fondation ARC nPJA 20151203337.

\section{Competing interests}

The authors declare no competing interests.

\section{References}

1. Doudna, J.A. and E. Charpentier. 2014. Genome editing. The new frontier of genome engineering with CRISPR-Cas9. Science 346:1258096.

2. Hazoumé, A., K. Naderi, E. Candolfi, C. Kedinger, B. Chatton, and M. Vigneron. 2011. A genetic analysis of Plasmodium falciparum RNA polymerase II subunits in yeast. Mol. Biochem. Parasitol. 176:127130.

3. Stoimenov, I. and T. Helleday. 2009. PCNA on the crossroad of cancer. Biochem. Soc. Trans. 37:605-613.

4. Baldeck, N., R. Janel-Bintz, J. Wagner, A. Tissier, R.P. Fuchs, P. Burkovics, L. Haracska, E. Despras, et al. 2015. FF483-484 motif of human Pol eta mediates its interaction with the POLD2 subunit of Pol delta and contributes to DNA damage tolerance. Nucleic Acids Res. 43:2116-2125.

5. Ran, F.A., P.D. Hsu, J. Wright, V. Agarwala, D.A. Scott, and F. Zhang. 2013. Genome engineering using the CRISPR-Cas9 system. Nat. Protoc. 8:22812308.

6. Hoege, C., B. Pfander, G.-L. Moldovan, G. Pyrowolakis, and S. Jentsch. 2002. RAD6-dependent DNA repair is linked to modification of PCNA by ubiquitin and SUMO. Nature 419:135-141.

7. Wang, S.-C., Y. Nakajima, Y.-L. Yu, W. Xia, C.-T. Chen, C.-C. Yang, E.W. Mclntush, L.-Y. Li, et al. 2006. Tyrosine phosphorylation controls PCNA function through protein stability. Nat. Cell Biol. 8:1359-1368.

8. Yoshimi, K., Kunihiro, Y., Kaneko, T., Nagahora, H,. Voigt, B. and Mashimo T. 2016. ssODNmediated knock-in with CRISPR-Cas for large genomic regions in zygotes. Nat Commun. 7:10431.

Received 06 October 2016; accepted 08 December 2016.

Address correspondence to Marc Vigneron, Université de Strasbourg, UMR7242 Biotechnologie et Signalisation Cellulaire, Ecole Supérieure de Biotechnologie de Strasbourg, CS10413, 67412 Illkirch, Cedex, France. E-mail: marc.vigneron@unistra.fr

To purchase reprints of this article, contact: biotechniques@fosterprinting.com 